\title{
Estimation of Chromatic Dispertion with FR. FT in Optical Cable
}

\author{
M.Ravi Shankar, A.Sreenivas
}

\begin{abstract}
The Telecom operators are in a great pressure to accommodate their optical back bone to migrate to new mobile generation. One of the most impact parameter to restrict the propagating distance is the Chromatic Dispersion. So in order to avoid the effect estimate the cause. With these lines, in our paper we have introduced a novel technique to estimate CD using FrFT in the optical fiber cable [2]. The technique involves in the scanning the chirping order for determining the $C D$. In this method cumulative CD in the optical media can be estimated shall be less than $80 \mathrm{ps} / \mathrm{nm} / \mathrm{km}$ and total CD less than $30 \mathrm{~ns} / \mathrm{nm}$
\end{abstract}

Keywords: OF Cable, Chromatic Dispersion, Fr.FT, Single Mode OF

\section{INTRODUCTION}

Now Telcos transforming all the e-commerce solutions converges with the mobile digital data with the advancing their present mobile generation. In this scenario the $\mathrm{CD}$ is the most seriously affecting the propagating signal distance to one tenth with advancing the speed of data network backbone ie MPLS [2]. The mitigation of the CD requires real time manipulation of the estimated CD [12]. The accumulated dispersion in the fiber channel can be compensated up to $10000 \mathrm{ps} / \mathrm{nm}$ [4] for the present optical DWDM network. But internet backboned with the MPLS cloud involves real time estimation of the CD. This is the real time challenge of the network engineer Hence Fr FT is the estimation technique for CD in the MPLS based DWDM packet network [5].

FrFT is the broad sub category of STFT and Weiner Transform [8] and these transforms can be easily understood in the time frequency (TF) plan. Fr FT is the mother of all the Fourier Transforms and the chirping parameter can be represented as a transform angle in the Time Frequency plan. Hence FrFT is the important tool for the analysis with the help of combination of time and frequency plans.

In [1] delineated various methods for the estimation of Dispersion using Fractional Fourier Transform. The implemented algorithm is the novice model which works efficiently in the independent of media and the modulation Techniques. This algorithm deals with an OF signal propagated in the simulated optical media. The output has been treated with the Fr FT algorithm. The algorithm involves the digitization of the Fr FT which involves robust computation efficiency as par that of the conventional FT.

\section{OPERATING PRINCEPLE}

The Fr. FT is the broad category of TF transform as that of Short Time Fourier Transform. As shown in the Fig. 1, the

. Revised Manuscript Received on December 13, 2019.

* Correspondence Author

M.Ravisankar*, JTO, BSNL, Hyderabad, Telangana, India

Dr.A.Sreenivas, Professor,Department of ECE,GITAM University, Visakhapatnam. mutual complementary pair of the time signal and the transformed frequency signals respectively with the angle $\alpha$ which infers the $C D$ or chirping signal to calculate the parameter $\mathrm{p}$ and can be refers to $(2 * \alpha) /$ pi [1]. FrFT of a signal can be expressed with $F \alpha(u)$ and defined as [2]

The FrFT is special category of TF transform as that of Short Time Fourier pair. In the trailing figure 1 it can be related the pair in the FT environment for the time and frequency. The rotation of the new time plan with reference to the new frequency can be expressed as the chirping angle $\alpha$ and the LFM parameter is the important data for obtaining the $\mathrm{p}$ of the signal can be equal to $\left(2^{*} \alpha\right) / \mathrm{pi}$ [1].

FrFT of the signal can be expressed with $F \propto(u)$ and defined as [2].

FrFT formulae

$$
\begin{aligned}
& F \alpha(u)=\int_{-\infty}^{\infty} f(t) K \alpha(t, u) d t \\
& \text { And } \\
& F(t)=\int_{-\infty}^{\infty} F(u) K \alpha(t, u) d u
\end{aligned}
$$

Kernel equation $\quad \mathrm{K}(\mathrm{t}, \mathrm{u})=$

$$
\left\{\begin{array}{c}
\sqrt{((1-j \cot \alpha) / 2 \pi)} \exp \left(j \frac{(\mathrm{t} 2+\mathrm{u} 2)}{2} \cot \alpha-\frac{\mathrm{jtu}}{\text { sina }}\right) \\
\text { if } \alpha \text { is not a multiple of } \pi \\
\delta(\mathrm{t}-\mathrm{u}) \\
\delta(\mathrm{t}+\mathrm{u})
\end{array}\right.
$$

Here $\mathrm{p}=(2 * \alpha) / \pi$

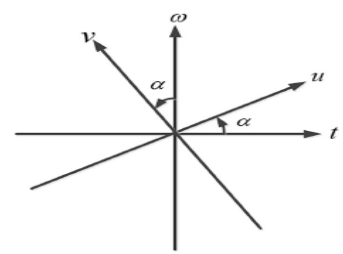

Fig. 1 Frequency Time plan and its LFT form for $\alpha$.

We can conclude that the LFM parameter directly proportion to the time axis. The $\mathrm{x}$-axis represents the real time and related to the $y$ - axis of frequency plan and they are directly related by an chirping projection of $\alpha$.

Input LFM optical signal can be expressed as

$$
s(t)=M \exp (i(2 \pi f t+D+\pi L t 2)
$$

Where $M$ represents maximum strength of input parameter $\mathrm{f}$ is frequency of the modulating optical variable, $\mathrm{D}$ represents the constant value and is the $\mathrm{L}$ is the LFM vaiable [1]. The $s(t)$ represented as

$$
\begin{aligned}
\mathrm{S} \alpha(\mathrm{u})= & \sqrt{((1-j \cot \alpha) / 2 \pi) \int_{-\infty}^{\infty} \mathrm{A}} \mathrm{A} \exp \\
& \left(\mathrm{j}\left[\frac{(\cot \alpha)}{2}+\pi \mathrm{C}\right) \mathrm{t} 2\right.
\end{aligned}
$$




\section{Estimation of Chromatic Dispertion with FR. FT in Optical Cable}

$$
\left.+(2 \pi f-\operatorname{ucsc} \alpha) t+\left(\frac{1}{2} u 2 \cot \alpha+D\right)\right]
$$

The equation (4) can be used for finding the maximum value that resulted from the scanning for the optimum order, while setting the (4) to null, $s(t)$ becomes an impulse response and that obtained hence $\mathrm{p}$ optimum of the system. Make chirping component set to zero order can call optimum order [1]

$$
\frac{(\cot \alpha)}{2}+\pi \mathrm{C}=0
$$

$\rightarrow$ Order becomes $-\frac{2}{\pi} * \arctan \left(\frac{1}{2 \pi \mathrm{c}}\right)$

Since order is $(2 * \alpha) / \mathrm{pi}$

\section{$2 \pi f-$ ucsc $\alpha=0$ implies $\quad \mathrm{U}=2 \pi f \sin \alpha$}

Here $\mathrm{C}$ is the LFM parameter and signal can be visualized with an AF [1] in the guided media. So the guided wave represented as following equation [2]

$$
f(t)=M . e \frac{[1+j C j)}{2 T_{0}^{2}} t 2
$$

$$
\begin{aligned}
& \text { So } \quad f(u)=\quad \sqrt{((1-j \cot \alpha) / 2 \pi)} \\
& \text { G }\left[\left\{j \frac{\mathrm{t}^{2}}{2}\left(\cot \alpha-\frac{\mathrm{c}}{\mathrm{T}_{0}^{2}}\right)-\mathrm{jut} \csc \alpha\right\}-\frac{\mathrm{t}^{2}}{2 \mathrm{~T}_{0}^{2}} \quad \mathrm{~J}\right. \text { dt } \\
& \text { If } \cot \alpha=\frac{c}{W_{0}^{2} x} \text { So order p can be } \\
& \frac{2}{\pi} \cot ^{-1}\left(\frac{W_{0}^{2}}{c}\right)
\end{aligned}
$$
in [9].

Then the order can be obtained as in (10) with subsisting

$$
P_{0}-1=\frac{2}{\pi} \cot ^{-1}\left(\frac{W_{0}^{2}}{C}\right)
$$

\section{ALGORITHM DESIGN}

The following steps give the computation of digital FrFT algorithm .Equation (1) and (2) consists of double LFM multiplications and single LFM convolution [12]. The Complexity of $2 * \mathrm{~N}$ (as dual LFM multiplications) $+\mathrm{N}^{*} \cdot \log$ $(2 \mathrm{~N})$ (as two Discrete Fourier Transforms) $=\mathrm{N}^{*} \log (2 \mathrm{~N})$. Here $\mathrm{N}=2 \mathrm{P}+1$ and $\mathrm{P}$ is the total no. of sampling points. It is to be noted that one LFM convolution needs to Discrete Fourier Transform and can be expressed as (11) [4]

$$
\begin{aligned}
& f[n]=\left\{\begin{array}{cc}
\frac{1}{N} \sum_{k=0}^{N-1} f_{1}[k] e^{j(2 \pi / N) k n} & 0 \leq k \leq N-1 \\
0 & \text { else }
\end{array}\right. \\
& f_{1}[k]=\left\{\begin{array}{cc}
\sum_{n=0}^{N-1} f[n] e^{-j(2 \pi / N) k . n} & 0 \leq k \leq N-1 \\
0 & \text { else }
\end{array}\right.
\end{aligned}
$$

Equation (11) main part expressed as $F(k, n)$ and the equation (12) represents as

$$
\begin{aligned}
& f_{1}[k]= \begin{cases}\sum_{n=0}^{N-1} f[n] \cdot F(k, n) \quad 0 \leq k \leq N-1\end{cases} \\
& =\frac{1}{\sqrt{N}} \sum_{k=0}^{N-1} f_{1}[k] e^{-j(2 \pi \cdot k / N) n} 0 \leq k \leq N-1
\end{aligned}
$$

$$
\text { Where } F(k, n)=W^{(k / N)} / \sqrt{N} \text { \& } W=e^{-j(2 \pi / N)} \text {. }
$$

This can be expressed in the Eigen matrix (13).

$$
f_{1}=F \cdot f
$$

$$
F=E A E^{T}
$$

Here $\mathrm{F}$ decomposed into $\mathrm{E}$ orthogonal matrix and A Eigen matrix.

Next same design has to be incorporated for fractional Fourier Transform for the order of a then $f{ }$ can be expressed as [5]

$$
f_{a}=F^{a} . f
$$

Expression (13) further can be shown as $F=Z A^{a} Z^{T}$

Eigenvectors decomposition of DFRFT can be

$\mathbf{F}^{\alpha}=\left[\begin{array}{llll}\mathbf{d}_{\mathbf{0}} & \mathbf{d}_{\mathbf{1}} & \cdots & \mathbf{d}_{\mathrm{N}-1}\end{array}\right] \cdot\left[\begin{array}{ccccc}1 & 0 & \cdots & \cdots & 0 \\ 0 & e^{-j \cdot \alpha} & \ddots & & \vdots \\ \vdots & \ddots & \ddots & \ddots & \vdots \\ \vdots & & \ddots & \ddots & 0 \\ 0 & \cdots & \cdots & 0 & e^{-j \cdot \alpha(N-1)}\end{array}\right] \cdot\left[\begin{array}{c}\mathbf{d}_{\mathbf{0}}{ }^{\top} \\ \mathbf{d}_{1}{ }^{\mathbf{T}} \\ \vdots \\ \mathbf{d}_{\mathrm{N}-1}{ }^{\mathbf{T}}\end{array}\right]$

This is very simple and fast rule to generate the algorithm for FrFT but the total number of multiplications gone to cube time of $\mathrm{N}$. Even though the simple procedure encouraging but the estimation time for the fast fractional Fourier Transform will be discouraged with the motto of time constraint. So a new algorithm for finding the accumulated [8] CD with the help of the fast FrFT approach that consumes time to $\mathrm{N} \log \mathrm{N}$ times.

The FrFT (1) [12] can be divided into 3 stages

Multiplication of LFM signal

The convolution of the LFM

Proceeding with 2nd LFM multiply

With this procedure (1) can be written as (16)

$$
\left.\left.F_{\alpha}(u)=\sqrt{1-j \cot \alpha} / 2 \pi\right) \int f(t) \cdot \exp \left(\frac{j\left(t^{2}+u^{2}\right)}{2}\right) \cot \alpha-j t u / \sin \alpha\right) d t
$$

The Equation (16) some trigonometric transformation results

$\left.\frac{j\left(t^{2}+u^{2}\right)}{u^{2}}\right) \cot \alpha-\frac{j t u}{\sin \alpha}=t^{2}(\cot \alpha-\csc \alpha)$
$u^{2}(\csc \alpha)+(t-u)^{2} \csc \alpha+$ 
The resulting 3 tan functions can be drawn from (17) and for $y^{s}(\mathrm{x})$ becomes as

$$
y^{s}(\mathrm{x})=\mathrm{A}_{\phi} \int \exp \left(-j c s c \alpha\left(t^{2}-u^{2}\right)\right) \cdot f(t) \cdot \exp \left(-\frac{j \tan \alpha}{2} t^{2}\right) d t
$$

By placing (18) into (16)

$$
F_{\alpha}(u)=\sqrt{\frac{1-j \cot \alpha}{2 \pi}} \exp \left(-\frac{j \tan \alpha}{2} u^{2}\right) \int f(t) \cdot \exp \left(-j c s c \alpha\left(t^{2}-u^{2}\right)\right) \cdot \exp \left(-\frac{j \tan \alpha}{2} t^{2}\right) d
$$

LFM signal expressed with $y\left(t^{\prime}\right)$ so equation (19) results

$$
y^{\prime \prime}(\mathrm{x})=\mathrm{A}_{\phi} \int \exp \left(-j \beta\left(t^{2}-u^{2}\right)\right) \cdot y\left(t^{p}\right) \cdot d t^{t}
$$

The convolution formulae derived and represented with $h(t)$ so the impulse response can be

$y^{s}(\mathrm{t})=\mathrm{A}_{\phi} \int \mathrm{h}\left(\left(t-t^{p}\right)\right) \cdot y\left(t^{n}\right) \cdot d t^{t}$

Where $y\left(t^{\prime}\right)=f(t) \cdot \exp \left(-j \tan \left(\frac{\alpha}{2}\right) \cdot t^{2}\right) \cdot d t^{\prime}$

And $\quad \mathrm{h}(t)=\int_{\Delta x}^{\Delta x} H(v) \exp (2 \pi v t) d v$

And $H(v)=e^{j\left(\frac{\pi}{4}\right)} \exp \left(-\frac{i \pi v^{2}}{\beta}\right)$

$$
g^{g}\left(\frac{m}{2 \Delta t}\right)=\sum_{n=-N}^{N} h\left(\frac{m-n}{2 \Delta t}\right) \mathbf{g}\left(\frac{n}{2 \Delta t}\right)
$$

Thus this derivation (22) utilized for the fast Fr FT implementation in lines with theFFT algorithm can be visualized as

$$
\begin{aligned}
& f_{a}=F_{I}^{a} f \\
& F_{I}^{a}=D A H_{l p} A J .
\end{aligned}
$$

The input signal sampled with Nyquest rate twice that of bandwidth with $\frac{1}{2 * \sqrt{0 w}}$ with the signal bandwidth can be in the interval $\left[-2 \delta w_{s} 2 \delta w\right]$ and this digital processed to decrease the Bandwidth with decimation by a factor of $\mathrm{D}$ and after completing the FrFT algorithm the signal again interpolated with sampling by a up sampling factor of .

$\mathrm{A}$ is ranked to the diagonal elements hence the number of multiplications limited [12] and only corresponding to LFM multiplication, and is the convolved to $\mathrm{H}$ matrix limiting the estimation time.

FrFT has been implemented in the MATLAB for rectangular pulse as shown in given the figure 2.1 for order zero which results the same signal. The results checked with [2] and have been exactly tallied and the chirp pulse with its order has been postulated as in equations (1)(2).
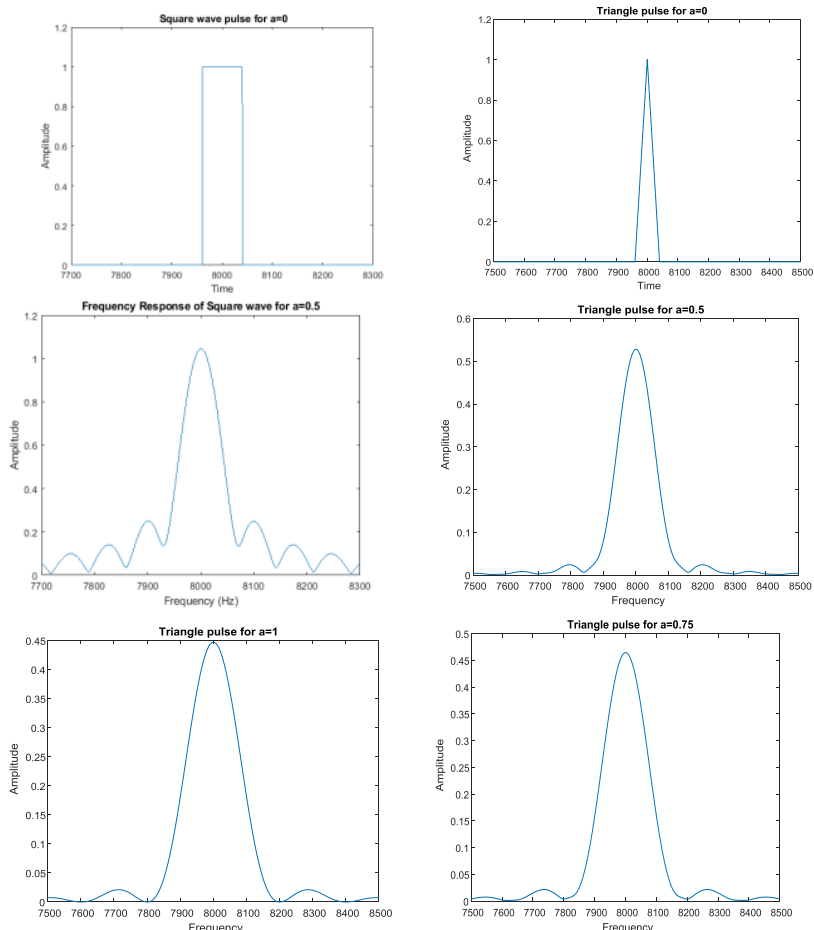

Fig. 2. Fast digital FrFT output for various orders.

Digital fast FrFT has been implemented for the rectangular pulse [13] and triangular pulse have tested and the results with various orders for these pulses order ranging from $0,0.5,0.75$ for triangular pulse with order zero replicates the input signal as shown in Fig 2.1 and 2., triangular pulse for order of one results doublesinc pulse as shown in the Fig 2.6 and sinc pulse for rectpuls shown in Fig 2.5.With the Fig 2 it can be inference that the with increasing the order from zero to one FT pulse tring to touch the time axis. With $\mathrm{a}=3$ inverse Fourier Transform resulted [13]. As the value of a increases beyond 0 to 4.the results will be limited and same as that appeared for the basic order limited to 0 to 4 [13]. The results here with tabulated with remarks for reference.

Table I: FrFT orders information

\begin{tabular}{|c|l|l|l|}
\hline S.NO & Property & Remarks \\
\hline 1 & 0 & Identity & $\begin{array}{c}\text { Output is } \\
\text { same as } \\
\text { input }\end{array}$ \\
\hline 2 & 0.2 & FrFT & \\
\hline 3 & 0.5 & FrFT & \\
\hline 4 & 0.75 & FrFT & $\begin{array}{c}\text { Fourier } \\
\text { Transform }\end{array}$ \\
\hline 5 & 1 & FT & \begin{tabular}{c} 
Inverse \\
Fourier \\
\hline 6
\end{tabular} \\
\hline & 3 & IFF & Transform \\
\hline
\end{tabular}




\section{Estimation of Chromatic Dispertion with FR. FT in Optical Cable}

\section{SIMULATION AND EXPERIMETAL RESULTS}

Chirp pulse attenuated below the $10 \mathrm{~dB}$ along with the data signal as in band Optical fiber System simulated model has been given as shown in Fig 3. The implemented algorithm expressed in [1][13]. The model is open with all the inline modulation models. Here we have worked with DP Pulse Code Keying and QAM analog modulation models.

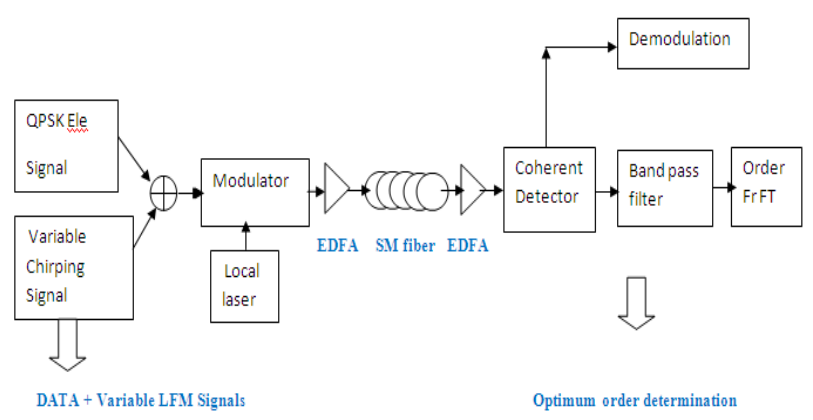

Fig 3. Estimation of CD with Chirping signal

We have implemented the model with OPTISYSTEM 13.0 for simulation of real time Single mode OFC system and characteristics of the cable varied and the $\mathrm{CD}$ estimated using MATLAB as shown in the Fig 4.The model checked Chromatic dispersion below $70 \mathrm{ps} / \mathrm{nm}$.

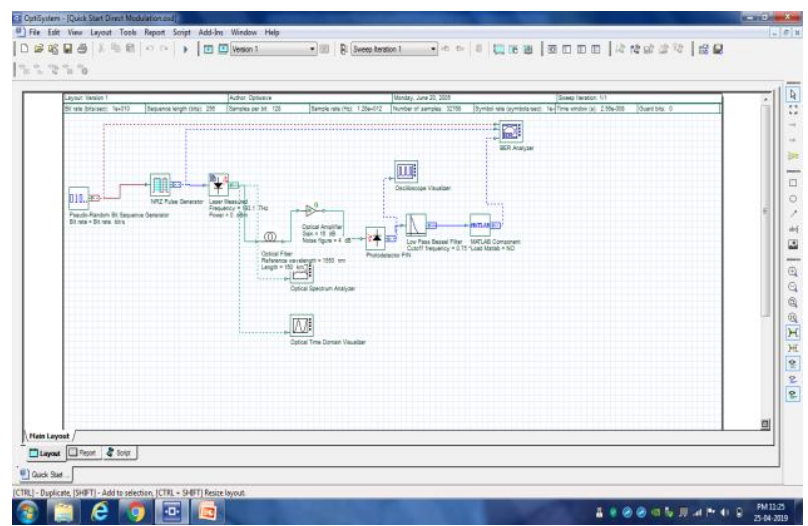

Fig 4. Algorithm design for FrFT estimation

The chirping or VLFM signal added along with the optical data signal with the fiber charectestics of attenuation made 0.2 $\mathrm{Db} / \mathrm{km}$ and non dispersion fiber. This made Eye diagram with in Fig 5.1 depicts that the even the attenuations can be compensated at the receiver with amplifier and so attenuation compensated. As the distance increased to $160 \mathrm{~km}$ as in Fig 5.2 , with $0.2 \mathrm{Db} / \mathrm{km}$ attenuation and non dispersion and the eye diagram shows that the original signal retrieved without distortion.

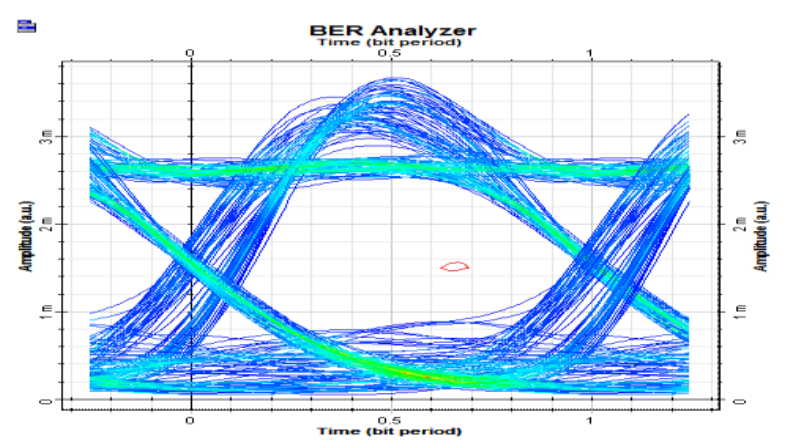

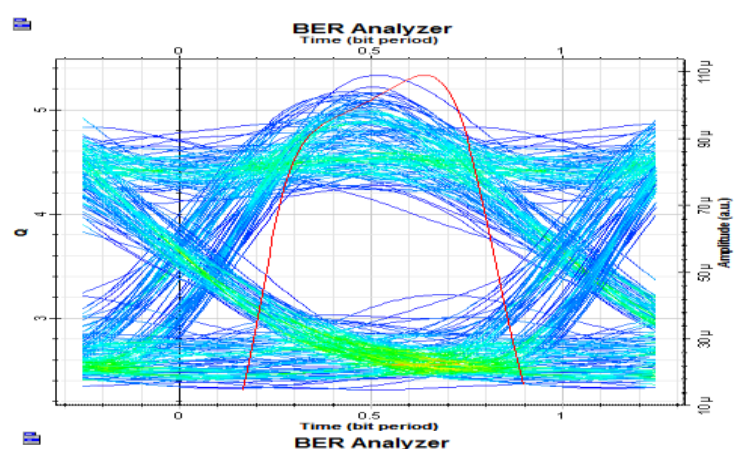
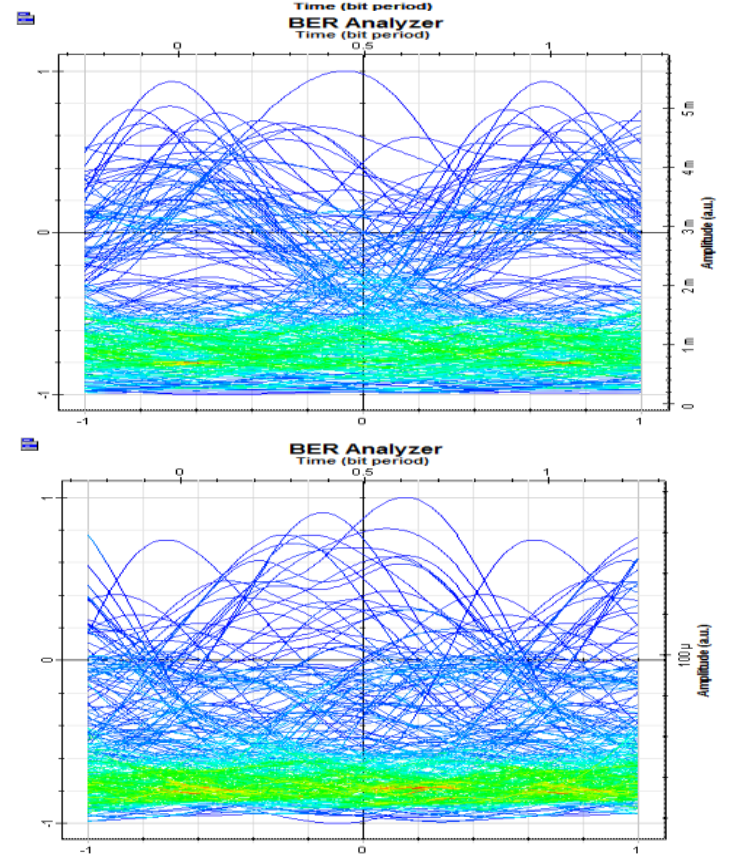

Fig. 5. EYE diagram results with different length, CD

Now for the Fig 5.3 we have made dispersion with at 16.75 $\mathrm{ps} / \mathrm{nm} / \mathrm{km}$ [11] then the received signal distorted and the eye closed with distortion for rest of the Fig 5 and at the receiver can not able to retrieve the input data. We have also experimentally simulated for the coherent generation and detection of the DP QPSK signal has been Simulated using opti system 13.0 as shown in the Fig 6 [13]. In this model cable length can be varied from $80 \mathrm{~km}$ to $1000 \mathrm{~km}$, attenuation and dispersion fixed at $0.2 \mathrm{~dB} / \mathrm{km}$ and $16.75 \mathrm{~nm} / \mathrm{ps} / \mathrm{km}$ [2][13] simultaneously and distortion results with increasing the length of the OF cable. Here the bandwidth speed fixed at 10GBPS. The length of optical fiber kept at $80 \mathrm{~km}$ and the constellation diagram shown in Fig 7

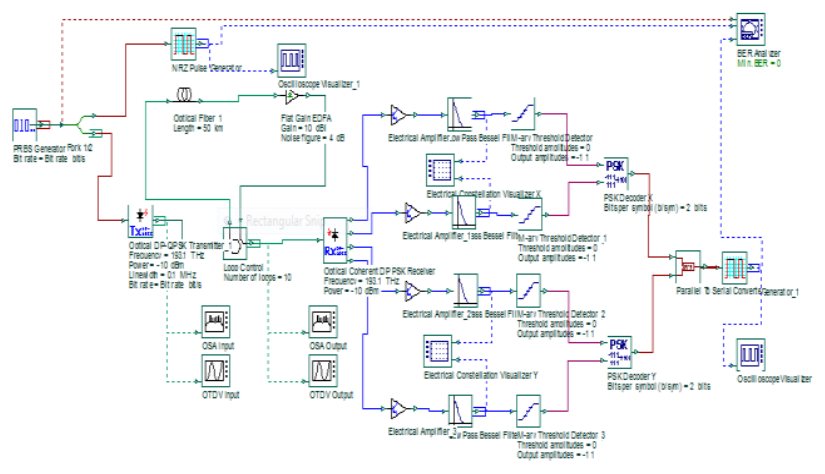

Fig .6. Transmitter and receiver of Dual Polarization QPSK with optisystem 13.0

Published By:

Blue Eyes Intelligence Engineering \& Sciences Publication 


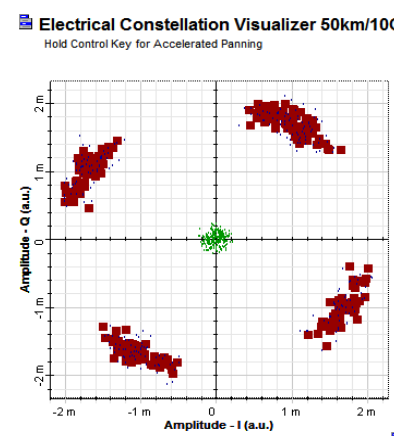

를Electrical Constellation Visualizer 100km/10G Hold Control Key for Accelerated Panning

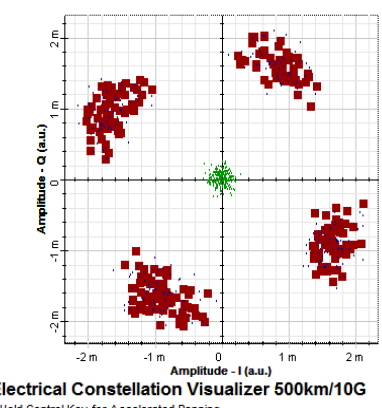
当Electrical Constellation Visualizer $100 \mathrm{~km} / 10 \mathrm{G}$ Electrical Constellation Visualizer $500 \mathrm{~km} / 10 \mathrm{G}$
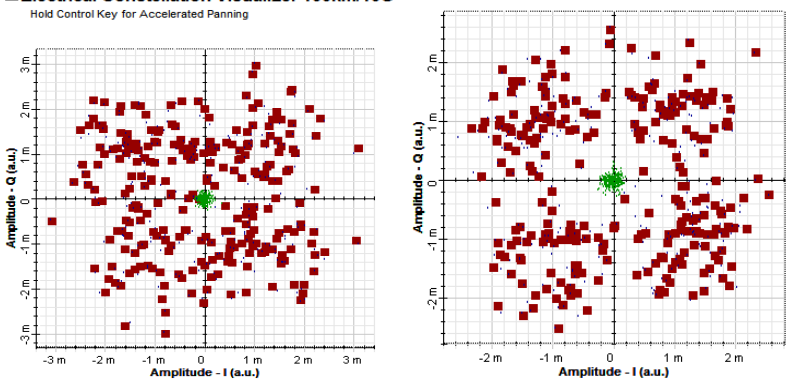

Fig. 7. Constellation diagram for Coherent detection of DPQPSK Signal results for 10 Gbps

\section{CONCLUSIONS AND FUTURE WORK}

Detected signal has been successfully recoved.In the second case with increasing the length to $100 \mathrm{~km}$ the signal dispersion increased and can be within the limitation and can be able to receive at the detector end. When the distance increased to $500 \mathrm{~km}$ the detector can able to recover at the threshold of $8425 \mathrm{~nm} / \mathrm{ps}$ for $500 \mathrm{~km}$ optical fiber .The signal has been Corrupted by the $600 \mathrm{~km}$ and scrambled at $1000 \mathrm{~km}$.

Present model has been implemented Fast digital Fractional Fourier Transform and tested with the rectangular pulse. The inband LFM signal has been modulated using DP-QPSK and QAM modulation with varying distance with fixed attenuation and dispersion. The detector end tested the data with mat lab and CD of the tested data and is exactly tallied with the output estimation of data. The Opti system simulated data to be tested with the [1] novel approach illustrated.

\section{REFERENCES}

1. M.Ravisankar, A.Sreenivas, "A Review on Estimation of Chromatic Dispersion using Fractional Fourier Transform in Optical Fiber Communication", 2018 International Conference on Smart Systems and Inventive Technology (ICSSIT), IEEE explore 2018.

2. G. P. Agrawal, Nonlinear Fiber Optics (5th ed.), New York: Academic, 2013.

3. Ozaktas, H.M., "Relationships among ray optical, Gaussian beam, and fractional Fourier transform descriptions of first-order optical systems", Optics Communications, 19971101.

4. Bultheel, A.. "Computation of the fractional Fourier transform", Applied and Computational Harmonic Analysis, 200405.

5. Billur Barshan, Birsel Ayrulu. "Fractional Fourier transform pre-processing for neural networks and its application to object recognition", Neural Networks, 2002.

6. Z. Pan, C. Yu, and A. E. Willner, "Optical performance monitoring for the next generation optical communication networks," Opt. Fiber Technol., vol. 16, no. 1, pp. 20-45, 2010.

7. A. Yang and X. Chen, "Method for measuring chromatic dispersion of optical fiber link through fractional order Fourier transformation,"
China Patent Application no. CN 201,410,752,087, Publication no. CN104467969, Mar. 25, 2015.

8. Sejdic, E.. "Time-frequency feature representation using energy concentration: An overview of recent advances", Digital Signal Processing, 200901

9. H. Zhou et al., "Fractional Fourier transformation-based blind chromatic dispersion estimation for coherent optical communications," J. Light wave Technol., vol. 34 , no. 10, pp. 1-1, May 2016.

10. Yang, Liu, Chen, "A FrFT based method for measuring chromatic dispersion and SPM in optical fibers" Optical Fiber Technology, Elsevier 34 pp 59 -64 2017

11. Sole-Pareta,Josep, Suresh Subramanian, Davide Careglio, and Salvatore "Cross-Layer Approaches for Planning and Operating Impairment-Aware Optical Networks", Proceedings of the IEEE, 2012

12. Lu, Yang, Chams Baker, Liang Chen, and Xiaoyi Bao. "Chromatic-Dispersion Monitor Based on a Differential Phase-Shift Method Exclude quotes Off Exclude bibliography Off Exclude matches Off Using a Kerr Phase-Interrogator", IEEE Photonics Journal, 2015.

13. [13] M.Ravisankar, A.Sreenivas, "CD Estimation using FrFT in SM OFC ", Vol 11,10-SI,2019.

\section{AUTHORS PROFILE}

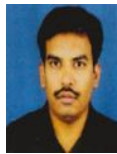

M.Ravisankar working as an executive in BSNL. He completed his B.E. from Andhra University 2002, M.E. from Osmania University 2008 . He is working as a scholar from GITAM University on the topic CD in Optical fiber. He is a member of of the International Association of Engineers.

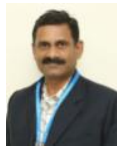

Dr. A.Sreenivas is a Professor in GITAM University, Visakhapatnam. He has vast experience and research in the field of Digital, Satellite Communications, and Embedded Systems. He is a member of Indian ScienceCongress Association. He has received the best achiever award in the year 2014 